\title{
UMA INTRODUÇÃO A SOMATÓRIOS FRACIONÁRIOS: APRENDENDO A SOMAR UMA QUANTIDADE NÃO INTEIRA DE PARCELAS
}

\author{
ÁGDA TALITA GALVÃO E JOCEMAR DE QUADROS CHAGAS
}

\begin{abstract}
RESUMO. Neste artigo apresentamos uma introdução à teoria de somatórios fracionários, que permite considerar somas finitas com quantidades não inteiras de parcelas. Este tipo de somatório tem seu primeiro registro em um exemplo devido a Euler, em uma publicação de 1755 [7], mas só voltou a ter vez na literatura em 2005 [18]. Expomos os axiomas, a definição de funções fracionário-somáveis e a fórmula fundamental para somatórios fracionários propostos por M. Müller e D. Schleicher, além de alguns exemplos, incluindo o exemplo de Euler. Como este artigo é, possivelmente, o primeiro registro em língua portuguesa sobre somatórios fracionários, excluída a dissertação [11], para facilitar a leitura do texto e a ambientação do leitor com os detalhes introduzidos nesta teoria, mostramos o passo-a-passo de muitos dos procedimentos realizados.
\end{abstract}

\section{INTRODUÇÃO}

Ao longo da história o conceito de número foi desenvolvido gradualmente. Inicialmente eram conhecidos apenas números naturais, depois números racionais, negativos, reais e complexos, introduzidos em alguma ordem conforme o decorrer da evolução da humanidade, quando surgiam necessidades, por exemplo, de contar animais, particionar objetos, registrar dívidas, etc.

É provável que a primeira operação matemática a surgir tenha sido a adição, surgida a partir da sistematização do processo natural de contagem. O modo como a contagem foi abstraída, junto com alguns dos sistemas usados para representar quantidades por meio de símbolos, os números, permitiram que algumas propriedades das quantidades fossem observadas, como por exemplo a possibilidade de efetuar de uma vez só a adição de dois ou mais objetos a um grupo, em vez de somar um objeto de cada vez. O entendimento

Data de aceitação: Setembro de 2020.

Palavras chave. Somas, Somatório, Somatório Fracionário, Parcelas Não Inteiras.

Autora 1, Ágda T. Galvão, agradece a SBM, IMPA, UEPG e UAB/CAPES pela oferta e manutenção do PROFMAT. 
da operação aditiva foi sendo constantemente aprimorado, por exemplo, com o estabelecimento das propriedades comutativa e associativa:

i) A adição é comutativa: $a+b=b+a$;

ii) A adição é associativa: $a+(b+c)=(a+b)+c$,

entre outras. A notação que usamos para escrever sinteticamente uma soma longa (com uma quantidade finita ou infinita de parcelas) utiliza o símbolo $\sum$. Nos referimos a esta forma de denotar um somatório como notação sigma, pois "Euler estabeleceu a notação de somatório que usamos hoje, usando sigma, uma letra grega maiúscula, para o simbolizar a soma." (ROSA, [22]). A origem e difusão desta notação são melhores pontuadas por Cajori ([3]):

O sinal de somatório é devido a Euler (1755), que diz: "summam indicabimus signo $\Sigma$ ”, ou seja, $\Sigma$ indica o sinal de soma. Este símbolo foi utilizado por Lagrange, mas, de resto, recebeu pouca atenção durante o Séc. XVIII. ‥ O símbolo $\Sigma$ para expressar "soma" aparece novamente em La Théorie Analytique de la chaleur de Fourier, publicada em 1822, e nas funções elípticas de Jacobi em 1829. Cauchy utilizou três índices $m, n, r$, como em $\sum_{m}^{n} r f r$.

Segundo Graham ([12]), a expressão $\sum_{k=1}^{n} f(k)$, onde $n$ é um número inteiro positivo, nos diz para incluir na soma precisamente aqueles termos $f(k)$ cujo índice $k$ é um inteiro que se encontra entre o menor e o maior limites 1 e $n$, inclusive. Por ser uma notação compacta, elegante, e por ter propriedades que facilitam operações algébricas, a notação sigma para somatórios é muito importante em vários campos da matemática, bem como em outras áreas como estatística, física, química, etc.

Quando são considerados somatórios com um número infinito de parcelas, conhecidos por séries numéricas (cuja notação traz o símbolo $\infty$ no limite superior do somatório), inicialmente deve-se decidir se a série é convergente, e se for, é interessante tentar calcular seu valor numérico, sendo importante conhecer o comportamento das sequências envolvidas (ver, e.g., [17]).

Estes conceitos (somatório clássico e séries numéricas) estão bem claros para os matemáticos da atualidade, e encontram-se inseridos em livros didáticos dos mais variados níveis de estudo, na maioria das vezes usando a notação sigma para somatórios diretamente (ou seja, sem uma seção prévia introduzindo a notação), indicando que ela é considerada pelos autores de tais livros como sendo de conhecimento pleno por todos (e é muito provável que seja).

Mas que significado podemos atribuir a um somatório do tipo $\sum_{\nu=1}^{x} f(\nu)$, quando $x \in \mathbb{R}$ ou $\mathbb{C}$ ?

Passamos agora a olhar para somatórios onde os índices são de ordens não-inteiras, chamados índices de ordens arbitrárias, que abrangem índices racionais, reais e até mesmo complexos, aos quais convencionou-se chamar índices fracionários (da mesma forma que na nomenclatura do cálculo fracionário - ver, e.g., [4], [14], [21]). Por exemplo, quais seriam as somas dos primeiros -7 números de uma sequência, ou dos $\pi$ primeiros termos 
da série harmônica? Este tipo de somatório vem sendo tratado, recentemente, com o nome de somatório de índices fracionários, ou apenas somatório fracionário ([1], [18]).

Müller e Schleicher ([20]) comentam que poderíamos pensar que um método para calcular este tipo de somas deveria ter sido descoberto há pelo menos duzentos anos, mas para surpresa desses autores (e nossa) uma tal teoria não parece ter sido investigada na literatura ou ser conhecida pelos especialistas antes de 2005 ([18]), além de breves observações esporádicas, nem mesmo no trabalho de Euler ([6], [7]), que nos brinda com um belo exemplo de uma soma com um número racional de termos, ao utilizar uma soma com um número não inteiro de termos como um dos métodos para introduzir funções. De fato, Euler apresentou o primeiro exemplo de somatório fracionário:

$$
\sum_{\nu=1}^{-1 / 2} \frac{1}{\nu}=-2 \ln (2) .
$$

No entanto, uma formulação adequada do problema e sua solução foram apresentadas apenas a partir de 2005 por Müller e Schleicher ([18], [19], [20]), que entre vários exemplos encontraram o interessante caso onde somatórios fracionários podem ser utilizados para obter a função gama, denotada por $\Gamma(x)$, e que em certo sentido estende a noção de fatorial de um número para os números reais (e complexos).

Como afirmam Tenreiro Machado et al. ([16]) os conceitos de "fracionário" embutidos nos conceitos de diferencial e de integral de funções permitem uma notável e frutífera generalização do sistema de operadores do cálculo clássico, e acreditamos que o mesmo ocorrerá com o conceito de somatório. A extensão do significado da inserção adequada de ordens fracionárias nos operadores clássicos da matemática pode ser comparada ao avanço que ocorreu na antiguidade quando surgiu a necessidade do conceito de números racionais, e na sequência e em momentos posteriores, dos irracionais e dos reais. $\mathrm{O}$ sucesso destas "novas" ferramentas em aplicações às ciências de alguma forma podem superar outras possíveis generalizações matemáticas e ajustar-se melhor à modelagem de fenômenos físicos, contribuindo assim para o desenvolvimento da fronteira da ciência, mas ainda é cedo para fazer a mensuração desta contribuição no caso dos somatórios fracionários.

Pretendemos, com base nos trabalhos de Müller e Schleicher ([18], [19], [20]) e de Euler ([6], [7]), apresentar em língua portuguesa os conceitos básicos da teoria para somatórios fracionários. Partiremos de um pequeno número de axiomas bem motivados, e apresentaremos uma definição única para somatórios com um número não inteiro de termos. Utilizando a definição, mostraremos exemplos de identidades dos somatórios clássicos que permanecem válidas no contexto dos somatórios fracionários, e além disso, apresentaremos dois exemplos de somas fracionárias que não encontram expressão equivalente para somatórios clássicos.

\section{SOMATÓRIOS FRACIONÁRIOS - OS AXIOMAS}

Consideraremos o contexto de funções complexas definidas em $\mathbb{C}$, isto é,

$$
\begin{aligned}
f: \mathbb{C} & \rightarrow \mathbb{C} \\
z & \mapsto f(z),
\end{aligned}
$$


onde se deseja somar quantidades não inteiras de termos deste tipo. Neste texto, $x, y, z \mathrm{e}$ $s$ são usados para representar números complexos, enquanto $f$ e $g$ representam funções complexas definidas em $\mathbb{C}$ ou seus subconjuntos, sujeitos a condições que serão especificadas na próxima seção.

A primeira necessidade é apresentar condições naturais para somas com um número complexo arbitrário de termos: os axiomas, que listamos a seguir.

Axioma (S1) - Continuidade da soma:

$$
\sum_{\nu=x}^{y} f(\nu)+\sum_{\nu=y+1}^{z} f(\nu)=\sum_{\nu=x}^{z} f(\nu) .
$$

Axioma (S2) - Invariância por translação:

$$
\sum_{\nu=x+s}^{y+s} f(\nu)=\sum_{\nu=x}^{y} f(\nu+s) .
$$

Axioma (S3) - Linearidade para constantes arbitrarias $\lambda, \mu \in \mathbb{R}$ :

$$
\sum_{\nu=x}^{y}(\lambda f(\nu)+\mu g(\nu))=\lambda \sum_{\nu=x}^{y} f(\nu)+\mu \sum_{\nu=x}^{y} g(\nu) .
$$

Axioma (S4) - Consistência com a definição clássica:

$$
\sum_{\nu=1}^{1} f(\nu)=f(1)
$$

Axioma (S5) - Somas de monômios:

Para cada $d \in \mathbb{N}$, a aplicação

$$
z \mapsto \sum_{\nu=1}^{z} \nu^{d}
$$

é holomórfica em $\mathbb{C}$ (funções holomorfas são funções definidas sobre um subconjunto aberto do plano complexo $\mathbb{C}$ com valores em $\mathbb{C}$ e que são diferenciáveis em cada ponto; ver e.g. [9]).

\section{Axioma (S6) - Continuidade no deslocamento à direita:}

Se $\lim _{n \rightarrow \infty} f(z+n)=0$ para cada ponto $z \in \mathbb{C}$, então

$$
\lim _{n \rightarrow \infty} \sum_{\nu=x}^{y} f(\nu+n)=0 .
$$

Mais geralmente, se existe uma sequência de polinômios $\left(p_{n}\right)_{n \in \mathbb{N}}$ de grau fixo, tal que $\left|f(z+n)-p_{n}(z+n)\right| \rightarrow 0$ quando $n \rightarrow \infty$ para todo $z \in \mathbb{C}$, então vale

$$
\left|\sum_{\nu=x}^{y} f(\nu+n)-\sum_{\nu=x}^{y} p_{n}(\nu+n)\right| \longrightarrow 0 .
$$

Os primeiros quatro axiomas (S1) - (S4) são tão óbvios e necessários, que é difícil imaginar construir uma teoria para somatórios que os violem. Com esses quatro axiomas, 
facilmente pode ser verificado que vale

$$
\sum_{\nu=1}^{n} f(\nu)=f(1)+f(2)+\cdots+f(n)
$$

para cada $n \in \mathbb{N}$, de forma que, ao considerar esses quatro axiomas, fica estabelecida a consistência com a definição clássica de soma.

$\mathrm{O}$ axioma (S5) é motivado pelas conhecidas fórmulas

$$
\sum_{\nu=1}^{n} \nu=\frac{n(n+1)}{2} ; \sum_{\nu=1}^{n} \nu^{2}=\frac{n(n+1)(2 n+1)}{6} ; \sum_{\nu=1}^{n} \nu^{3}=\left(\frac{n(n+1)}{2}\right)^{2} ;
$$

e fórmulas similares para potências superiores. Se respeitamos os axiomas (S1) - (S5), todas essas fórmulas permanecem válidas ao considerarmos valores $n \in \mathbb{C}$ arbitrários.

Finalmente, o axioma (S6) também pode ser apresentado como uma condição natural. No caso mais simples (2) está expresso o fato que, se $f$ tende a zero quando seu argumento tende ao infinito, então a soma sobre "o domínio limitado" $[x, y]$ deve fazer o mesmo. Em (3) o mesmo se aplica, apenas é acrescentada uma aproximação polinomial para a função $f$.

Para uma grande classe de funções $f$, há uma maneira única de definir um somatório $\sum_{\nu=1}^{z} f(\nu) \operatorname{com} z \in \mathbb{C}$ que respeita a todos os axiomas (S1) - (S6). Na próxima seção iremos apresentar tal definição, e denotaremos tais somas, os somatórios fracionários, com o símbolo $\sum_{\nu=1}^{z} f(\nu)$.

\section{SOMATÓRIOS FRACIONÁRIOS - UMA DEFINIÇÃO ÚNICA}

Para ganhar experiência no uso dos axiomas (S1) - (S6) para o manejo de somas e começar a compreender como tais axiomas podem ser usados para determinar de forma única um método para somar uma quantidade não inteira de termos, começaremos somando polinômios. O caso mais simples é uma soma do tipo $\sum_{\nu=1}^{x} c$, onde $c \in \mathbb{C}$ é constante $\mathrm{e}$ $x \in \mathbb{R}$.

Como um primeiro exemplo, vamos somar $\sum_{\nu=1}^{1 / 2} c$. Se consideramos o axioma (S1) válido, podemos escrever

$$
\sum_{\nu=1}^{1 / 2} c+\sum_{\nu=3 / 2}^{1} c=\sum_{\nu=1}^{1} c .
$$

Ao aplicarmos o axioma (S2) na segunda parcela do lado esquerdo desta equação, e o axioma (S4) no lado direito, obtemos

$$
\sum_{\nu=1}^{1 / 2} c+\sum_{\nu=1}^{1 / 2} c=c
$$


e assim

$$
\sum_{\nu=1}^{1 / 2} c=\frac{c}{2}
$$

Por linearidade, este procedimento simples pode ser estendido para definir qualquer soma, com um número racional de termos, de polinômios.

O lema 3.1, a seguir, será necessário na proposição 3.2 para a definição de soma fracionária de uma função polinomial (soma com quantidade de parcelas racional, real, ou mesmo complexa).

Lema 3.1: Para qualquer polinômio $p: \mathbb{C} \rightarrow \mathbb{C}$, de grau $m$, existe um único polinômio $P: \mathbb{C} \rightarrow \mathbb{C}$ tal que $P(0)=0$ e $P(z)-P(z-1)=p(z)$ para todo $z \in \mathbb{C}$.

Demonstração: Fixado qualquer polinômio $p: \mathbb{C} \rightarrow \mathbb{C}$ de grau $m$, para $n=1,2, \cdots, m+$ 1 definimos $\alpha_{n}=\sum_{i=1}^{n} p(i)$ e consideramos os $m+2$ pontos $(0,0)$ e $\left(n, \alpha_{n}\right)$. Pelo teorema de interpolação de Lagrange (ver, e.g., [5], [13]), existe um único polinômio $P$ de grau máximo $m+1$ que passa por esses $m+2$ pontos, isto é, com $P(0)=0$ e $P(n)=\alpha_{n}$ para $1 \leq n \leq m+1$, tal que $P(n)-P(n-1)=p(n)$.

Tomamos agora $q: \mathbb{C} \rightarrow \mathbb{C}$ definido por $q(z)=P(z)-P(z-1)$; o grau de $q$ é no máximo $m$, e vale $q(n)=P(n)-P(n-1)=p(n)$ para $1 \leq n \leq m+1$. Segue portanto que $q=p$, ou seja, $P(z)-P(z-1)=p(z)$, para todo $z \in \mathbb{C}$ (em particular, o grau do polinômio $P$ é $m+1)$.

Apresentamos, a seguir, a definição para a soma fracionária de uma função polinomial.

Proposição 3.2: Para qualquer polinômio $p: \mathbb{C} \rightarrow \mathbb{C}$, seja $P: \mathbb{C} \rightarrow \mathbb{C}$ o único polinômio tal que $P(0)=0$ e $P(z)-P(z-1)=p(z)$ para todo $z \in \mathbb{C}$. Então:

(I) A definição de soma fracionária de polinômios dada por

$$
\sum_{\nu=x}^{y} p(\nu):=P(y)-P(x-1)
$$

satisfaz os axiomas (S1) a (S6).

(II) Inversamente, toda teoria de soma que satisfaça aos axiomas (S1), (S2), (S3), e (S4) também deve satisfazer a definição (4) para todo polinômio p e para todo $x, y \in \mathbb{C}$ com diferença racional, i.e., com $y-x \in \mathbb{Q}$.

(III) Toda teoria de soma que satisfaça aos axiomas (S1), (S2), (S3), (S4) e (S5) também deve satisfazer a definição (4) para todo polinômio p e para todo $x, y \in \mathbb{C}$.

Demonstração: Dado um polinômio $p: \mathbb{C} \rightarrow \mathbb{C}$, para provar a afirmação (I), tomamos o único polinômio $P$ como no lema 3.1. 
Para verificar que o axioma (S1) é satisfeito pela eq. (4), calculamos:

$$
\begin{aligned}
\sum_{\nu=x}^{z} p(\nu)+\sum_{\nu=z+1}^{y} p(\nu) & =P(z)-P(x-1)+P(y)-P((z+1)-1) \\
& =P(y)-P(x-1) \\
& =\sum_{\nu=x}^{y} p(\nu) .
\end{aligned}
$$

Para verificar que o axioma (S4) é satisfeito pela eq. (4), calculamos:

$$
\sum_{\nu=1}^{1} p(\nu)=P(1)-P(1-1)=P(1)-P(0)=p(1) .
$$

Para verificar que o axioma (S5) é satisfeito pela eq. (4), basta notar que para cada $d \in \mathbb{N}$, existe um único polinômio $P: \mathbb{C} \rightarrow \mathbb{C}$ tal que $P(0)=0$ e $P(z)-P(z-1)=z^{d}$, de forma que podemos escrever

$$
\sum_{\nu=1}^{z} \nu^{d}=P(z)-P(1-1)=P(z)-P(0)=P(z)
$$

para qualquer $z \in \mathbb{Z}$, e polinômios são holomorfos em $\mathbb{C}$ (ver, e.g., [9]).

Para verificar que o axioma (S2) é satisfeito pela eq. (4), consideramos o polinômio $p$, e para cada $s \in \mathbb{C}$, definimos os polinômios $\widetilde{p}, \widetilde{P}: \mathbb{C} \rightarrow \mathbb{C}$ respectivamente por $\widetilde{p}(z):=p(z+s)$ e $\widetilde{P}(z):=P(z+s)-P(s)$, de forma que o polinômio $\widetilde{P}$ satisfaz $\widetilde{P}(0)=0$ e $\widetilde{P}(z)-\widetilde{P}(z-1)=\widetilde{p}(z)$. De fato:

$$
\begin{aligned}
\widetilde{P}(0) & =P(0+s)-P(s)=0 ; \mathrm{e} \\
\widetilde{P}(z)-\widetilde{P}(z-1) & =P(z+s)-P(s)-(P(z-1+s)-P(s)) \\
& =P(z+s)-P(z+s-1) \\
& =p(z+s) \\
& =\widetilde{p}(z) .
\end{aligned}
$$

Segue portanto

$$
\begin{aligned}
\sum_{\nu=x}^{y} p(\nu+s) & =\sum_{\nu=x}^{y} \widetilde{p}(\nu) \\
& =\widetilde{P}(y)-\widetilde{P}(x-1) \\
& =(P(y+s)-P(s))-(P(x-1+s)-P(s)) \\
& =P(y+s)-P(x+s-1) \\
& =\sum_{\nu=x+s}^{y+s} p(\nu) .
\end{aligned}
$$

Para verificar que o axioma (S3) é satisfeito pela eq. (4), consideramos $\lambda, \mu \in \mathbb{R}$, o polinômio $p$, um outro polinômio $q: \mathbb{C} \rightarrow \mathbb{C}$ e o único polinômio $Q: \mathbb{C} \rightarrow \mathbb{C}$ que satisfaz a $Q(0)=0$ e $Q(z)-Q(z-1)=q(z)$ para todo $z \in \mathbb{C}$, conforme o lema 3.1. 
Definindo o polinômio $r: \mathbb{C} \rightarrow \mathbb{C}$ por $r(z):=\lambda p(z)+\mu q(z)$ para todo $z \in \mathbb{C}, \mathbf{o}$ polinômio $R: \mathbb{C} \rightarrow \mathbb{C}$ definido por $R(z):=\lambda P(z)+\mu Q(z)$ para todo $z \in \mathbb{C}$ satisfará $R(z)-R(z-1)=r(z)$ e $R(0)=0$. De fato:

$$
\begin{gathered}
R(0)=\lambda P(0)-\lambda Q(0)=\lambda .0-\mu .0=0-0=0 ; \mathrm{e} \\
R(z)-R(z-1)=(\lambda P(z)+\mu Q(z))-(\lambda P(z-1)+\mu Q(z-1)) \\
=(\lambda P(z)-\lambda P(z-1))+(\mu Q(z)-\mu Q(z-1)) \\
=\lambda(P(z)-P(z-1))+\mu(Q(z)-Q(z-1)) \\
=\lambda p(z)+\mu q(z)=r(z) .
\end{gathered}
$$

Então, calculamos:

$$
\begin{aligned}
\sum_{\nu=x}^{y}(\lambda p(\nu)+\mu q(\nu)) & =\sum_{\nu=x}^{y} r(\nu) \\
& =R(y)-R(x-1) \\
& =(\lambda P(y)+\mu Q(y))-(\lambda P(x-1)+\mu Q(x-1)) \\
& =(\lambda P(y)-\lambda P(x-1))+(\mu Q(y)-\mu Q(x-1)) \\
& =\lambda(P(y)-P(x-1))+\mu(Q(y)-Q(x-1)) \\
& =\lambda \sum_{\nu=x}^{y} p(\nu)+\mu \sum_{\nu=x}^{y} q(\nu) .
\end{aligned}
$$

Para verificar que o axioma (S6) é satisfeito pela eq. (4), consideramos o espaço linear $V_{m}$ de polinômios complexos de grau menor ou igual a $m \in \mathbb{N}$, e ao definirmos $\|p\|:=$ $\sum_{i=0}^{m}|p(i)|$ para $p \in V_{m}$, introduzimos uma norma em $V_{m}$. Definimos então o operador linear $\sum_{x}^{y}: V_{m} \rightarrow \mathbb{C}$ através de $\sum_{x}^{y} p:=\sum_{\nu=x}^{y} p(\nu)$. Como $\operatorname{dim}\left(V_{m}\right)=m+1<\infty$, o operador $\sum_{x}^{y}$ é limitado (e, portanto, contínuo).

Assim, se $\left(q_{n}\right)_{n \in \mathbb{N}} \subset V_{m}$ é uma sequência de polinômios que converge para zero na norma $\|\cdot\|$ ao $n \rightarrow \infty$, isto é, se $\lim _{n \rightarrow \infty}\left\|q_{n}\right\|=0$, vale a convergência $\lim _{n \rightarrow \infty} \sum_{x}^{y} q_{n}=0$ em $\mathbb{C}$.

Nesse contexto, a convergência constante na afirmação (3) do axioma (S6) é obtida ao considerarmos a sequência de polinômios $\left(q_{n}\right)_{n \in \mathbb{N}}$ dada por $q_{n}(z):=p(z+n)-p_{n}(z+n)$ e observarmos que a convergência pontual de $\left|q_{n}(z)\right|$ para zero, dada como hipótese no axioma (S6), implica na convergência para zero da sequência $q_{n}$ na norma $\|\cdot\| \mathrm{e}$, por seguinte, na convergência para zero de $\sum_{x}^{y} q_{n}$ em $\mathbb{C}$, isto é,

$$
\lim _{n \rightarrow \infty}\left(\sum_{\nu=x}^{y} p(z+n)-\sum_{\nu=x}^{y} p_{n}(z+n)\right)=0 .
$$

Obs.: Para a verificação do axioma (S6) foram necessários conceitos de análise funcional, que podem ser consultados em [2]. 
Para provarmos a afirmação (II), supomos que existe alguma definição que permite obter a soma fracionária $\sum_{\nu=x}^{y} p(\nu)$ e que respeite os axiomas $(\mathrm{S} 1)$ - (S4), e estendemos a ideia que usamos para mostrar que $\sum_{\nu=1}^{1 / 2} c=\frac{c}{2}$. Tomamos inteiros $r, s \geq 1$ e, usando o axioma (S1), podemos escrever

$$
\sum_{\nu=1}^{r} \nu^{d}=\sum_{\nu=1}^{\frac{r}{s}} \nu^{d}+\sum_{\nu=\frac{r}{s}+1}^{\frac{2 r}{s}} \nu^{d}+\ldots+\sum_{\nu=\frac{(s-2) r}{s}+1}^{\frac{(s-1) r}{s}} \nu^{d}+\sum_{\nu=\frac{(s-1) r}{s}+1}^{r} \nu^{d}
$$

onde o lado esquerdo é um somatório clássico, que sabemos determinar o valor. Usando os axiomas (S2) e (S3), podemos rearranjar o lado direito como segue:

$$
\begin{aligned}
& \sum_{\nu=1}^{r} \nu^{d}=\sum_{\nu=1}^{\frac{r}{s}} \nu^{d}+\sum_{\nu=\frac{r}{s}+1}^{\frac{r}{s}+\frac{r}{s}} \nu^{d}+\sum_{\nu=\frac{2 r}{s}+1}^{\frac{r}{s}+\frac{2 r}{s}} \nu^{d}+\cdots+\sum_{\nu=\frac{(s-1) r}{s}+1}^{\frac{r}{s}+\frac{(s-1) r}{s}} \nu^{d} \\
& =\sum_{\nu=1}^{\frac{r}{s}}(\nu+0)^{d}+\sum_{\nu=1}^{\frac{r}{s}}\left(\nu+\frac{r}{s}\right)^{d}+\sum_{\nu=1}^{\frac{r}{s}}\left(\nu+\frac{2 r}{s}\right)^{d}+\cdots+\sum_{\nu=1}^{\frac{r}{s}}\left(\nu+\frac{(s-1) r}{s}\right)^{d} \\
& =\sum_{k=1}^{s-1} \sum_{\nu=1}^{\frac{r}{s}}\left[\left(\nu+\frac{k r}{s}\right)^{d}\right] \\
& =\sum_{k=1}^{s-1} \sum_{\nu=1}^{\frac{r}{s}}\left[\left(\nu+\frac{k r}{s}\right)^{d}+\nu^{d}-\nu^{d}\right] \\
& =\sum_{k=1}^{s-1} \sum_{\nu=1}^{\frac{r}{s}} \nu^{d}+\sum_{k=1}^{s-1} \sum_{\nu=1}^{\frac{r}{s}}\left[\left(\nu+\frac{k r}{s}\right)^{d}-\nu^{d}\right] \\
& =s \sum_{\nu=1}^{\frac{r}{s}} \nu^{d}+\sum_{k=1}^{s-1} \sum_{\nu=1}^{\frac{r}{s}} q_{d-1, k}(\nu)
\end{aligned}
$$

onde os polinômios $q_{d-1, k}(\nu)=\left(\nu+\frac{k r}{s}\right)^{d}-\nu^{d}$ tem grau $d-1$ (e observamos que $\left.q_{-1, k} \equiv 0\right)$.

Agora argumentamos por indução. Se $d=0$, a equação anterior determina claramente o valor de $\sum_{\nu=1}^{r / s} 1$. Por linearidade, fica também determinada a soma de constantes arbitrárias $c \in \mathbb{C}$ de 1 até $r / s$. Ao supormos que o valor da soma de qualquer polinômio de grau $d-1$ é bem determinado, a igualdade anterior também permite encontrar o valor de $\sum_{\nu=1}^{r / s} \nu^{d} \mathrm{e}$, por linearidade, concluímos ser possível determinar a soma de cada polinômio de grau $d$. 
Então, usamos o axioma (S2) para escrever

$$
\sum_{\nu=x}^{y} p(\nu)=\sum_{\nu=x-(x-1)+(x-1)}^{y-(x-1)+(x-1)} p(\nu)=\sum_{\nu=1+(x-1)}^{y-x+1+(x-1)} p(\nu)=\sum_{\nu=1}^{(y-x+1)} p(\nu+x-1)
$$

e concluímos que os axiomas (S1) - (S4) nos permitem determinar o valor da soma $\sum_{\nu=x}^{y} p(\nu)$ para todo $x, y \in \mathbb{C}$ tais que $y-x \in \mathbb{Q}$.

Como visto na demonstração da afirmação (I), a equação (4) é uma possível definição que satisfaz os axiomas (S1) - (S4); então, usamos (5) e (4) para escrever

$$
\sum_{\nu=1}^{(y-x+1)} p(\nu+x-1)=\sum_{\nu=x}^{y} p(\nu)=P(y)-P(x-1),
$$

e concluir que (4) é a única definição possível para $y-x \in \mathbb{Q}$.

Finalmente, para concluir a demonstração da afirmação (III), basta notar que a restrição $y-x \in \mathbb{Q}$ pode ser retirada ao supormos que a definição para obter a soma fracionária $\sum_{\nu=x}^{y} p(\nu)$ respeite os axiomas (S1) - (S4) e também o axioma (S5). A holomorfia é necessária apenas para $y-x \in \mathbb{C}$, pois para $y-x \in \mathbb{R}$ a equação (4) já será satisfeita ao exigirmos apenas continuidade no axioma (S5).

Para estabelecer uma definição de soma fracionária para uma classe mais ampla de funções $f: \mathbb{C} \rightarrow \mathbb{C}$, tudo o que necessitamos exigir sobre a função $f$ é que os valores de $f(n+z)$ possam ser aproximados por alguma sequência de polinômios $p_{n}(n+z)$, de grau fixo, quando $n \rightarrow+\infty$, além de um pequeno cuidado com os domínios de definição $U$ para o somatório fracionário: é necessária a propriedade

$$
z \in U \quad \Rightarrow \quad z+1 \in U \text {. }
$$

Convencionando que o polinômio nulo é o único polinômio de grau $-\infty$, apresentamos a seguinte definição:

Definição 3.3: Dados $U \subset \mathbb{C}$ e $m \in \mathbb{N} \cup\{-\infty\}$, uma função $f: U \rightarrow \mathbb{C}$ será chamada fracionário-somável de grau $m$ se forem satisfeitas as seguintes condições:

(i) $x+1 \in U$ para todo $x \in U$;

(ii) Existe uma sequência de polinômios $\left(p_{n}\right)_{n \in \mathbb{N}}$ de grau $m$ fixo tal que, para todo $x \in U$, ocorre

$$
\left|f(n+x)-p_{n}(n+x)\right| \rightarrow 0 \text { quando } n \rightarrow+\infty ;
$$

(iii) Para todo $x, y \in U$, existe o limite

$$
\lim _{n \rightarrow \infty}\left(\sum_{\nu=n+x}^{n+y} p_{n}(\nu)+\sum_{\nu=1}^{n}(f(\nu+x-1)-f(\nu+y))\right),
$$

onde a soma $\sum p_{n}$ é definida como em (4). 
Se uma função $f$ for fracionário-somável, usaremos a notação

$$
\sum_{\nu=x}^{y} f(\nu) \quad \text { ou, brevemente, } \sum_{x}^{y} f,
$$

e vale a fórmula fundamental do somatório fracionário:

$$
\sum_{\nu=x}^{y} f(\nu)=\sum_{\nu=1}^{\infty}(f(\nu+x-1)-f(\nu+y)) .
$$

Além disso, podemos definir produtório fracionário por

$$
\prod_{\nu=x}^{y} f(\nu):=\exp \left(\sum_{\nu=x}^{y} \ln (f(\nu))\right)
$$

sempre que $\ln (f)$ for fracionário-somável.

A definição 3.3 não depende da escolha dos polinômios $\left(p_{n}\right)_{n \in \mathbb{N}}$ que aproximam $f$. De fato, se $\left(\widetilde{p}_{n}\right)_{n \in \mathbb{N}}$ é outra escolha de polinômios que aproximam $f$, então vale

$$
\lim _{n \rightarrow \infty}\left(p_{n}(n+x)-\widetilde{p}_{n}(n+x)\right)=0, \quad \forall x \in U
$$

(e portanto para todo $x \in \mathbb{C}$ ), pois o conjunto de polinômios de grau máximo $m$ é um espaço linear de dimensão finita. Como visto na proposição 2.2, somas de polinômios satisfazem o axioma (S6), então, substituindo $f$ por 0 e $p_{n}$ por $\left(\widetilde{p}_{n}-p_{n}\right)$ na expressão (3), conclui-se facilmente que

$$
\lim _{n \rightarrow \infty}\left(\sum_{\nu=n+x}^{n+y} p_{n}(\nu)-\sum_{\nu=n+x}^{n+y} \widetilde{p}_{n}(\nu)\right)=0 .
$$

A soma fracionária apresentada na definição 3.3 é a única que satisfaz os axiomas (S1) - (S6), como mostra o teorema a seguir:

Teorema 3.4: A definição 3.3, de funções fracionário-somáveis, atende todos os axiomas (S1) - (S6) para domínios de definição adequados, e a fórmula fundamental do somatório fracionário (6) é a única que satisfaz aos axiomas (S1) - (S6) para a classe de funções considerada.

Demonstração: Para mostrar que a fórmula fundamental do somatório fracionário (6) é a única possível para somas fracionárias satisfazendo a todos os axiomas (S1) - (S6), consideramos uma soma do tipo $\sum_{\nu=x}^{y} f(\nu) \operatorname{com} x, y \in \mathbb{C}$, e, com o uso do axioma (S1), escrevemos

$$
\sum_{\nu=x}^{y} f(\nu)+\sum_{\nu=y+1}^{y+n} f(\nu)=\sum_{\nu=x}^{y+n} f(\nu)=\sum_{\nu=x}^{x+n-1} f(\nu)+\sum_{\nu=x+n}^{y+n} f(\nu),
$$

onde $n \in \mathbb{N}$ é arbitrário, e reorganizamos os termos como segue:

$$
\sum_{\nu=x}^{y} f(\nu)=\sum_{\nu=x}^{x+n-1} f(\nu)-\sum_{\nu=y+1}^{y+n} f(\nu)+\sum_{\nu=x+n}^{y+n} f(\nu)
$$




$$
\begin{aligned}
& =\sum_{\nu=1+(x-1)}^{n+(x-1)} f(\nu)-\sum_{\nu=1+(y)}^{n+(y)} f(\nu)+\sum_{\nu=x+n}^{y+n} f(\nu) \\
& =\sum_{\nu=1}^{n}(f(\nu+x-1)-f(\nu+y))+\sum_{\nu=x}^{y} f(\nu+n) .
\end{aligned}
$$

Na última linha da eq. (8) o primeiro somatório envolve um número inteiro de termos e pode ser avaliado classicamente. O problema a resolver fica então restrito à última soma do lado direito, onde o domínio da soma aparece deslocado $n$ unidades para a direita. Como a eq. (8) vale para cada inteiro $n$, podemos passar a eq. (8) ao limite com $n \rightarrow \infty$, e então usar o axioma (S6): se $f(n+z) \rightarrow 0$ quando $n \rightarrow \infty$ para todo $z$, então o axioma (S6) implica que o limite quando $n \rightarrow \infty$ na última soma da eq. (8) desaparece, e ficamos apenas com

$$
\sum_{\nu=x}^{y} f(\nu)=\sum_{\nu=1}^{\infty}(f(\nu+x-1)-f(\nu+y))
$$

que apresentamos em (6) como a fórmula fundamental do somatório fracionário.

Resta provar que a fórmula fundamental do somatório fracionário (6) realmente satisfaz todos os axiomas (S1) - (S6).

Claramente, os axiomas (S3) e (S5) são automaticamente satisfeitos.

Para provar que os axiomas (S1), (S2) e (S4) são satisfeitos, basta substituir a soma $\sum_{x}^{y} f(\nu)$ pela fórmula fundamental do somatório fracionário (6) no respectivo axioma, e fazer alguns poucos cálculos diretos. Por exemplo, substituindo a fórmula fundamental do somatório fracionário no lado esquerdo do axioma (S1), obtemos o lado direito:

$$
\begin{aligned}
\sum_{\nu=x}^{y} f(\nu) & +\sum_{\nu=y+1}^{z} f(\nu)= \\
= & \sum_{\nu=1}^{\infty}(f(\nu+x-1)-f(\nu+y))+\sum_{\nu=1}^{\infty}(f(\nu+y+1-1)-f(\nu+z)) \\
= & \sum_{\nu=1}^{\infty}((f(\nu+x-1)-f(\nu+y))+(f(\nu+y)-f(\nu+z))) \\
= & \sum_{\nu=1}^{\infty}(f(\nu+x-1)-f(\nu+z)) \\
= & \sum_{\nu=x}^{z} f(\nu) .
\end{aligned}
$$

Para provar que o axioma (S6) é satisfeito, utilizamos a fórmula fundamental do somatório fracionário (6) junto com os outros axiomas, e buscamos avaliar

$$
\lim _{n \rightarrow \infty}\left(\sum_{\nu=x}^{y} f(\nu+n)-\sum_{\nu=x}^{y} p_{n}(\nu+n)\right)
$$


da seguinte forma:

$$
\begin{aligned}
& \lim _{n \rightarrow \infty}\left(\sum_{\nu=x}^{y} f(\nu+n)-\sum_{\nu=x}^{y} p_{n}(\nu+n)\right)= \\
& =\lim _{n \rightarrow \infty}\left(\sum_{\nu=x}^{y} f(\nu+n)-\sum_{\nu=x}^{y} p_{n}(\nu+n)\right. \\
& \left.-\sum_{\nu=1}^{n}(f(\nu+x-1)-f(\nu+y))+\sum_{\nu=1}^{n}(f(\nu+x-1)-f(\nu+y))\right) \\
& =\lim _{n \rightarrow \infty}\left(\sum_{\nu=x}^{y} f(\nu+n)-\sum_{\nu=x}^{y} f(\nu+n)\right. \\
& \left.-\sum_{\nu=1}^{n}(f(\nu+x-1)-f(\nu+y))+\sum_{\nu=1}^{n}(f(\nu+x-1)-f(\nu+y))\right) \\
& =\lim _{n \rightarrow \infty}\left(\sum_{\nu=x}^{y} f(\nu+n)-\sum_{\nu=x}^{y} f(\nu)+\sum_{\nu=1}^{n}(f(\nu+x-1)-f(\nu+y))\right) \\
& =\lim _{n \rightarrow \infty}\left(\sum_{\nu=x}^{y} f(\nu+n)-\sum_{\nu=x}^{y} f(\nu)+\sum_{\nu=1}^{n} f(\nu+x-1)-\sum_{\nu=1}^{n} f(\nu+y)\right) \\
& =\lim _{n \rightarrow \infty}\left(\sum_{\nu=x+n}^{y+n} f(\nu)-\sum_{\nu=x}^{y} f(\nu)+\sum_{\nu=x}^{x+n-1} f(\nu)-\sum_{\nu=y+1}^{y+n} f(\nu)\right) \\
& =\lim _{n \rightarrow \infty}\left(\sum_{\nu=x}^{x+n-1} f(\nu)+\sum_{\nu=x+n}^{y+n} f(\nu)-\sum_{\nu=x}^{y} f(\nu)-\sum_{\nu=y+1}^{y+n} f(\nu)\right) \\
& =\lim _{n \rightarrow \infty}\left(\sum_{\nu=x}^{y+n} f(\nu)-\sum_{\nu=x}^{y+n} f(\nu)\right)=0 .
\end{aligned}
$$

Isso completa a demonstração de que a fórmula fundamental do somatório fracionário (6), para uma função $f: \mathbb{C} \rightarrow \mathbb{C}$ atendendo às condições dadas na definição 3.3, satisfaz a todos os axiomas (S1) - (S6).

Na próxima seção apresentamos alguns exemplos de somas fracionárias obtidas com a aplicação da definição 3.3 e da fórmula fundamental do somatório fracionário (6).

\section{Alguns EXEMPLOS DE SOMAS FRACIONÁRIAS}

Agora que conhecemos uma definição adequada para funções fracionário-somáveis e uma fórmula que permite obter o valor da soma de uma quantidade não inteira de termos (definição 3.3 e fórmula (6)), é natural a curiosidade por conhecer algumas das propriedades satisfeitas pelos somatórios clássicos (finitos) que permanecem válidas com essa abordagem mais geral, e também por conhecer algumas outras identidades que não encontram equivalência no caso clássico. 
Uma das mais básicas propriedades para somas finitas é a soma da série geométrica, que permanece válida para somatórios fracionários. De fato, considerando $0 \leq q<1$ por simplicidade, como a função $f(z)=q^{z}$ é "aproximadamente zero" para valores grandes de $z$, e vale $\lim _{n \rightarrow \infty} q^{z+n}=0$ para todo $z \in \mathbb{C}$, concluímos que $q^{z}$ é fracionário-somável, e, usando a fórmula fundamental do somatório fracionário (6), obtemos:

$$
\sum_{\nu=0}^{z} q^{\nu}=\sum_{\nu=1}^{\infty}\left(q^{\nu-1}-q^{\nu+z}\right)=\left(1-q^{z+1}\right) \sum_{\nu=1}^{\infty} q^{\nu-1}=\frac{1-q^{z+1}}{1-q}
$$

ou seja, a conhecida fórmula para a soma da série geométrica permanece válida para todo $z \in \mathbb{C}$.

No próximo exemplo, consideramos uma função cujo comportamento assintótico não se aproxima de zero, e usamos somatórios e produtórios fracionários para extrapolar a função fatorial clássica para todo $z \in \mathbb{C}$, exceto $z \in\{0,-1,-2,-3, \cdots\}$. Inicialmente, reescrevemos a eq. (8) para a função $f(z)=\ln (z)$ :

$$
\sum_{\nu=x}^{y} \ln (\nu)=\sum_{\nu=1}^{n}(\ln (\nu+x-1)-\ln (\nu+y))+\sum_{\nu=x}^{y} \ln (\nu+n)
$$

onde, para o último termo da eq.(9), notamos que para $z \in[x, y] \subset \mathbb{R}^{+}$, os valores $\ln (z+n)$ são relativamente bem aproximados pela função constante $\ln (n)$, com um erro que tende a zero quando $n \rightarrow \infty$ (dizemos que $f=\ln$ é "aproximadamente constante" quando seu argumento tende ao infinito). Então, usando o axioma (S3), podemos escrever

$$
\sum_{\nu=x}^{y} \ln (\nu+n)=\sum_{\nu=x}^{y} \ln n+\sum_{\nu=x}^{y}(\ln (\nu+x)-\ln (n))
$$

para cada $n \in \mathbb{N}$. Na eq. (10), a primeira soma do lado direito tem somando constante e pode ser avaliada através da eq. (4), e, pelo axioma (S6), a última soma se anula quando $n \rightarrow \infty$. Assim, considerando $\ln (n)$ constante antes de passar a eq. (10) ao limite, podemos concluir que

$$
\lim _{n \rightarrow \infty} \sum_{\nu=x}^{y} \ln (\nu+n)=\ln (n) \sum_{\nu=x}^{y} 1=(y-x+1) \ln (n) .
$$

Portanto, ao passar a eq. (9) ao limite $\operatorname{com} n \rightarrow \infty$, obtemos

$$
\sum_{\nu=x}^{y} \ln (\nu)=\lim _{n \rightarrow \infty}\left(\sum_{\nu=1}^{n}(\ln (\nu+x-1)-\ln (\nu+y))+(y-x+1) \ln n\right),
$$

de onde segue que

$$
\exp \left(\sum_{\nu=1}^{z} \ln \nu\right)=\lim _{n \rightarrow \infty}\left(\exp \left(\sum_{\nu=1}^{n}(\ln (\nu)-\ln (\nu+z))+z \ln n\right)\right) .
$$


Então, tomando a liberdade de escrever $z$ ! para $z \in \mathbb{R}$ ou $z \in \mathbb{C}$, e usando a definição de produtório fracionário dada na eq. (7), com $\nu$ no lugar de $f(\nu)$, calculamos

$$
\begin{aligned}
z ! \equiv \prod_{\nu=1}^{z} \nu & =\exp \left(\sum_{\nu=1}^{z} \ln (\nu)\right) \\
& =\lim _{n \rightarrow \infty}\left(n^{z} \exp \sum_{\nu=1}^{n} \ln \left(\frac{\nu}{\nu+z}\right)\right) \\
& =\lim _{n \rightarrow \infty}\left(n^{z} \cdot \prod_{\nu=1}^{n}\left(\frac{\nu}{\nu+z}\right)\right) \\
& =\Gamma(z+1) ;
\end{aligned}
$$

obtendo assim a função gama, que, para $z \in \mathbb{C} \backslash\{0,-1,-2,-3, \cdots\}$, é definida por

$$
\Gamma(z):=\int_{0}^{\infty} e^{-t} t^{z-1} d t
$$

e tem a seguinte representação na forma de limite (ver, e.g., [14] ou [21]):

$$
\Gamma(z)=\lim _{n \rightarrow \infty} \frac{n ! n^{z}}{z(z+1) \cdots(z+n)},
$$

utilizada na última passagem para obter a eq. (11), junto com a propriedade $z \Gamma(z)=$ $\Gamma(z+1)$.

Outra propriedade que permanece válida para somas fracionárias é a expansão da série binomial. Para recuperá-la, inicialmente lembramos que se $\alpha$ e $j$ são naturais, vale

$$
\left(\begin{array}{l}
\alpha \\
j
\end{array}\right)=\frac{\alpha !}{j !(\alpha-j) !},
$$

mas se $\alpha$ e $j \in \mathbb{C}$, então vale (ver [14] ou [21]):

$$
\left(\begin{array}{c}
\alpha \\
j
\end{array}\right)=\frac{\Gamma(\alpha+1)}{\Gamma(j+1) \Gamma(\alpha-j+1)} .
$$

Consideramos então $x \in \mathbb{C}$, com $|x|<1$, e $\alpha \in \mathbb{C} \backslash\{-1,-2,-3, \ldots\}$ e, usando a fórmula fundamental do somatório fracionário (6) para a função

$$
f(\nu)=\left(\begin{array}{l}
\alpha \\
\nu
\end{array}\right) z^{\nu}
$$

obtemos

$$
\sum_{\nu=0}^{x}\left(\begin{array}{l}
\alpha \\
\nu
\end{array}\right) z^{\nu}=\sum_{\nu=1}^{\infty}\left(\left(\begin{array}{c}
\alpha \\
\nu-1
\end{array}\right) z^{\nu-1}-\left(\begin{array}{c}
\alpha \\
\nu+x
\end{array}\right) z^{\nu+x}\right) .
$$

Então fazemos $x=\alpha$ na eq. (12). Em seu último termo ficamos com

$$
\left(\begin{array}{c}
\alpha \\
\nu+\alpha
\end{array}\right)=\frac{\Gamma(\alpha+1)}{\Gamma(\nu+\alpha+1) \Gamma(-\nu+1)},
$$


e como para todo $\nu \in \mathbb{N}^{*}$ vale $\Gamma(1-\nu)= \pm \infty$ e isso ocorre no último termo do somatório no lado direito da eq. (12), sempre teremos "algo" do tipo $\frac{\Gamma(\alpha+1)}{ \pm \infty}$, que deve ser considerado nulo. Assim, a eq. (12) pode ser reescrita como

$$
\begin{aligned}
\sum_{\nu=0}^{\alpha}\left(\begin{array}{l}
\alpha \\
\nu
\end{array}\right) z^{\nu} & =\sum_{\nu=1}^{\infty}\left(\begin{array}{c}
\alpha \\
\nu-1
\end{array}\right) z^{\nu-1} \\
& =(1+z)^{\alpha}
\end{aligned}
$$

onde aparece a expressão expandida da série binomial (ver [8], [15]).

Apresentamos a seguir duas novas identidades obtidas com o uso de somatórios e produtórios fracionários, e que não encontram alguma expressão equivalente com somatórios clássicos.

Com auxílio das seguintes identidades da função gama

$$
\Gamma(z) \Gamma(1-z)=\frac{\pi}{\operatorname{sen}(\pi z)}=\frac{i \pi}{\sinh (\pi i z)}
$$

(ver [21]) e da expressão da função gama em termos de um produtório fracionário obtida na eq. (11), podemos obter a primeira nova identidade:

$$
\tanh (\pi)=\prod_{\nu=1}^{-1 / 2}\left(\nu^{2}+1\right)
$$

A prova de (13), por ser um pouco mais trabalhosa, não será apresentada neste artigo, mas o leitor interessado pode acompanhar o desenvolvimento passo-a-passo em [11].

Para a segunda nova identidade, que apresentamos como exemplo final neste artigo, começamos por considerar a série harmônica. A função $f(z)=z^{-1}$, que pode ser considerada "aproximadamente zero" para $z$ grande, é fracionária-somável e podemos, portanto, aplicar a fórmula fundamental do somatório fracionário (6), obtendo:

$$
\sum_{\nu=1}^{x} \frac{1}{\nu}=\sum_{\nu=1}^{\infty}\left(\frac{1}{\nu+1-1}-\frac{1}{\nu+x}\right)=\sum_{\nu=1}^{\infty}\left(\frac{1}{\nu}-\frac{1}{\nu+x}\right)
$$

e, em particular:

$$
\begin{aligned}
\sum_{\nu=1}^{-1 / 2} \frac{1}{\nu} & =\sum_{\nu=1}^{\infty}\left(\frac{1}{\nu}-\frac{1}{\nu-\frac{1}{2}}\right)=\sum_{\nu=1}^{\infty}\left(\frac{1}{\nu}-\frac{2}{2 \nu-1}\right) \\
& =(1-2)+\left(\frac{1}{2}-\frac{2}{3}\right)+\left(\frac{1}{3}-\frac{2}{5}\right)+\left(\frac{1}{4}-\frac{2}{7}\right)+\cdots \\
& =(-2+1)+\left(-\frac{2}{3}+\frac{1}{2}\right)+\left(-\frac{2}{5}+\frac{1}{3}\right)+\left(-\frac{2}{7}+\frac{1}{4}\right)+\cdots \\
& =(-2+1)+\left(-\frac{2}{3}+\frac{2}{4}\right)+\left(-\frac{2}{5}+\frac{2}{6}\right)+\left(-\frac{2}{7}+\frac{2}{8}\right)+\cdots \\
& =-2\left(1-\frac{1}{2}+\frac{1}{3}-\frac{1}{4}+\frac{1}{5}-\frac{1}{6}+\frac{1}{7}-\frac{1}{8}+\frac{1}{9}-\frac{1}{10}+\cdots\right) \\
& =-2 \ln (2) .
\end{aligned}
$$


Recuperamos, assim, o resultado (1), isto é,

$$
\sum_{\nu=1}^{-1 / 2} \frac{1}{\nu}=-2 \ln 2,
$$

o primeiro exemplo de um somatório fracionário, obtido e anunciado por Euler em 1755 [7]. Como uma curiosidade final, destacamos que a soma

$$
1-\frac{1}{2}+\frac{1}{3}-\frac{1}{4}+\frac{1}{5}-\frac{1}{6}+\frac{1}{7}-\frac{1}{8}+\frac{1}{9}-\frac{1}{10}+\cdots
$$

é a expansão em série de MacLaurin da função $\ln (x)$, avaliada em $x=2$.

\section{CONSIDERAÇÕES FINAIS}

Apresentamos, neste texto, uma introdução em língua portuguesa à expansão da teoria concernente à mais básica das operações matemáticas: a adição. Somatórios clássicos, usados para somar uma quantidade finita de termos, cuja notação sigma foi introduzida por Euler (e.g., [6]) e difundida a partir de seu uso por Fourier [10], são considerados conhecidos por todos, em maior ou menor profundidade. Somatórios infinitos de termos reais, conhecidos por séries numéricas, são ensinados em nossos cursos de graduação e alguns casos até no ensino médio. A grande "novidade" introduzida por M. Müller e D. Schleicher ([18], [19], [20]) mas já apresentada, de alguma forma, por Euler [7] é a possibilidade de efetuar somas finitas contendo uma quantidade não inteira de parcelas. Com o advento da teoria para somatórios fracionários (consistente com a teoria para somatórios clássicos) passa a ser permitido somar sobre uma quantidade racional, real e mesmo complexa de termos.

Em um primeiro contato com esta teoria, é comum pensar que se trata de uma espécie de "soma contínua", talvez um pouco semelhante com o somatório infinitesimal costumeiramente apresentado como definição da integral definida; alertamos que isso é uma falácia, como o axioma (S1) induz a compreender. Quando consideramos um somatório do tipo

$$
\sum_{\nu=x}^{y} f(\nu)
$$

onde $f$ é uma função fracionário-somável e $x, y \in U \subset \mathbb{R}$ são dois números bem determinados, tal soma tem uma quantidade finita de parcelas, onde (possivelmente) apenas uma parcela é fracionária, sendo as demais parcelas inteiras. Por exemplo, com o uso do axioma (S1) a expressão

$$
\sum_{\nu=1}^{3,5} f(v)
$$

pode ser reescrita em uma das seguintes formas:

$$
\sum_{\nu=1}^{2} f(v)+\sum_{3}^{3,5} f(v) \text { ou } \sum_{\nu=1}^{3} f(v)+\sum_{4}^{3,5} f(v)
$$


ou seja,

$$
f(1)+f(2)+\sum_{3}^{3,5} f(v) \text { ou } f(1)+f(2)+f(3)+\sum_{4}^{3,5} f(v),
$$

em ambas temos uma soma de uma quantidade inteira de parcelas do tipo $f(v)$ com uma parcela fracionária, ou seja, de "comprimento" menor que uma unidade. Quando $x, y \in U \subset \mathbb{C}$, ainda não está totalmente claro para os autores qual seria a natureza da soma $\sum_{x}^{y} f(\nu)$; a única certeza é que não se trata de uma soma de infinitas parcelas. Além dessa certeza, por enquanto apenas conseguimos conjecturar que existe algum tipo de "independência do caminho" da soma, pois a fórmula fundamental do somatório fracionário (6) considera explicitamente valores de $f$ com argumentos dependendo dos limites inferior e superior $x$ e $y$ do somatório fracionário (fato também percebido em outros cálculos rabiscados e ainda não publicados).

Acreditamos que esta teoria para somatórios fracionários, desenvolvida recentemente, guarda ainda belas propriedades e identidades surpreendentes a serem descobertas e talvez até encontre utilidade na modelagem de situações reais, talvez com um belo futuro no desenvolvimento tecnológico. Esperamos que este artigo desperte o interesse no leitor em buscar aprender mais sobre o tema, e fazer suas próprias especulações. Recomendamos aos interessados a leitura da dissertação [11], escrita em português, dos artigos [18], [19], e [20], escritos pelos autores que deram o passo inicial desta teoria, e do livro [1].

\section{REFERÊNCIAS}

[1] Alabdulmohsin, I. M. - Summability Calculus A comprehensive theory of fractional finite sums. Springer, Switzerland (2018).

[2] Botelho, G. M. A.; Pellegrino, D. M.; Teixeira, E. V. - Fundamentos de Análise Funcional. Textos Universitários, $2^{\mathrm{a}}$ ed., Editora SBM, Rio de Janeiro (2015).

[3] Cajori, F. - A History of Mathematical Notations. Dover Publications, New York (1993).

[4] Camargo, R. F.; Oliveira, E. C. - Cálculo Fracionário. Editora Livraria da Física, São Paulo (2015).

[5] Caminha, A. - Tópicos de Matemática Elementar Volume 6 - Polinômios. $2^{\mathrm{a}}$ ed., Editora SBM, Rio de Janeiro (2016).

[6] Euler, L. - Institutiones Calculi Differentialis cum eius usu in Analysi Finitorum ac Doctrina Serierum [E212]. Petropolitanae Academiae Sumptibus, (1755); Republicado em Opera Omnia, series 1, vol 10.

[7] Euler, L. - Dilucitationes in capita postrema calculi mei differentialis de funcionibus inexplicabilibus [E613]. Supplementum in [E212] (1755); republicado em Memoires de l'academie des sciences de St.-Petersbourg 4 (1813), 88-119; republicado em Opera Omnia, series 1, vol 16, 1-33.

[8] Euler, L. - Nova demonstratio qvod potestatvm Binomii Newtoniana etiam pro exponentibus fractis valeat [E637]. Nova Acta Academiae Scientiarvm Imperialis Petropolitanae, Tomvs V (1787), 52-58 (Mathematica).

[9] Fernandez, C. S.; Bernardes Jr., N. C. - Introdução às Funções de uma Variável Complexa. Textos Universitários, $5^{\mathrm{a}}$ ed., Editora SBM, Rio de Janeiro (2019).

[10] Fourier, J.-B. J. - La théorie analytique de la chaleur. Chez Firmin Didot, Père ed Fils, Paris (1822).

[11] Galvão, A. T. - Somas, somatórios e termos não inteiros. Dissertação de Mestrado (PROFMAT), UEPG, Ponta Grossa (2020).

[12] Graham, R. L.; Knuth, D. E.; Patashnik, O. - Concrete mathematics: a foundation for computer science. $2^{\mathrm{a}}$ ed., Addison-Wesley Publishing, New York (1994).

[13] Hefez, A.; Villela, M. L. T. - Polinômios e Equações Algébricas. Coleção PROFMAT, Editora SBM, Rio de Janeiro (2012). 
[14] Kobuchei, A.; Luchko, Y. (Eds.) - Handbook of Fractional Calculus with Applications, vol. 1: Basic Theory. De Gruyter, Berlin (2019).

[15] Leachenski, A. A. - Binômio de Newton com expoente negativo e fracionário. Dissertação de Mestrado (PROFMAT), UEPG, Ponta Grossa (2017).

[16] Tenreiro Machado, J.; Lopes, A. M.; Duarte, F. B.; Ortigueira, M. D.; Rato, R. T. - Rhapsody in Fractional. Fractional Calculus \& Applied Analysis, Vol. 17, No. 4 (2014), 1188-1214.

[17] Matos, M. P. - Séries e Equações Diferenciais. Ed. Ciência Moderna, Rio de Janeiro (2017).

[18] Müller, M.; Schleicher, D. - How to Add a Non-Integer Number of Terms, and How to Produce Unusual Infinite Summations. Journal of Computational and Applied Mathematics 178 1-2 (2005), 347-360.

[19] Müller, M.; Schleicher, D. - Fractional sums and Euler-like identities. Ramanujan J., 21 (2010). 347360.

[20] Müller, M.; Schleicher, D. - How to add a noninteger number of Terms: From axioms to new identities. The American Mathematical Monthly. Vol. 118, $\mathrm{n}^{\circ} 2$ (2011). 136-152.

[21] Podlubny, I. - Fractional differential equations: an introduction to fractional derivatives, fractional differential equations, to methods of their solution and some of their applications. Vol. 198, Academic Press, London (1999).

[22] Rosa, C. A. P. - História da ciência: a ciência moderna. $2^{\mathrm{a}}$ ed., FUNAG, Brasília (2012).

Departamento de Matemática e estatística, UePG, 84030-900, Ponta Grossa, PR

Email address: agdatg@gmail.com, jocemarchagas@uepg.br 